\title{
Homogeneous extremal function for a ball in $\mathbb{R}^{2}$
}

\author{
by MirosŁaw BARAN (Kraków)
}

\begin{abstract}
We point out relations between Siciak's homogeneous extremal function $\Psi_{B}$ and the Cauchy-Poisson transform in case $B$ is a ball in $\mathbb{R}^{2}$. In particular, we find effective formulas for $\Psi_{B}$ for an important class of balls. These formulas imply that, in general, $\Psi_{B}$ is not a norm in $\mathbb{C}^{2}$.
\end{abstract}

0. Introduction. Let $\mathcal{P}\left(\mathbb{C}^{n}\right)$ and $\mathcal{H}\left(\mathbb{C}^{n}\right)$ denote the set of polynomials of $n$ complex variables and the set of homogeneous polynomials of $n$ variables, respectively. We denote by $\mathcal{L}\left(\mathbb{C}^{n}\right)$ the Lelong class of plurisubharmonic functions $u$ in $\mathbb{C}^{n}$ with logarithmic growth: $u(z) \leq$ const $+\log (1+\|z\|)$.

An important role in pluripotential theory and approximation theory of many variables is played by two extremal functions introduced by Siciak (see [Si1]-[Si5]) and called Siciak's extremal function (or polynomial extremal function) $\Phi_{E}$ and Siciak's homogeneous extremal function $\Psi_{E}$, respectively:

$$
\begin{aligned}
& \Phi_{E}(z)=\sup \left\{|p(z)|^{1 / \operatorname{deg} p}: p \in \mathcal{P}\left(\mathbb{C}^{n}\right), \operatorname{deg} p \geq 1,\|p\|_{E} \leq 1\right\}, \quad z \in \mathbb{C}^{n}, \\
& \Psi_{E}(z)=\sup \left\{|p(z)|^{1 / \operatorname{deg} p}: p \in \mathcal{H}\left(\mathbb{C}^{n}\right), \operatorname{deg} p \geq 1,\|p\|_{E} \leq 1\right\}, \quad z \in \mathbb{C}^{n},
\end{aligned}
$$

where $E$ is a fixed compact subset of $\mathbb{C}^{n}$. It is well known (see [Si4], [Si5]) that

and

$$
\log \Phi_{E}(z)=V_{E}(z):=\sup \left\{u(z): u \in \mathcal{L}\left(\mathbb{C}^{n}\right),\left.u\right|_{E} \leq 0\right\}
$$

$$
\Psi_{E}(z)=\sup \left\{u(z): u \text { is homogeneous psh in } \mathbb{C}^{n},\left.u\right|_{E} \leq 1\right\} .
$$

If $E$ is a circular set, there is a simple relation between $\Phi_{E}$ and $\Psi_{E}$ (see [Si4]):

$$
\Phi_{E}(z)=\max \left(1, \Psi_{E}(z)\right)
$$

1991 Mathematics Subject Classification: 41A17, 32F05.

Key words and phrases: homogeneous extremal function, Cauchy-Poisson transform. Research partially suported by grant No. 2 PO3A 05708 from KBN (Committee for Scientific Research) of Poland and by the European Programme PECO of the French Government. 
In particular, if $B$ is a closed unit ball with respect to a norm $q$ in $\mathbb{C}^{n}$ then

$$
\Psi_{B}(z)=q(z), \quad z \in \mathbb{C}^{n}
$$

(see $[\mathrm{Si} 4])$.

The situation is much more complicated if $B$ is a ball in $\mathbb{R}^{n}$ with respect to a norm $q$. Here we treat $\mathbb{R}^{n}$ as a subset of $\mathbb{C}^{n}$ such that $\mathbb{C}^{n}=\mathbb{R}^{n}+i \mathbb{R}^{n}$. It is known (see [Si1], [D]) that if $B_{n}$ is the unit Euclidean ball in $\mathbb{R}^{n}$, then $\Psi_{B_{n}}(z)$ is equal to the Lie norm:

$$
\Psi_{B_{n}}(z)=L_{n}(z)=\left(\frac{\|z\|^{2}+\left|z^{2}\right|}{2}\right)^{1 / 2}+\left(\frac{\|z\|^{2}-\left|z^{2}\right|}{2}\right)^{1 / 2},
$$

where $z^{2}=z_{1}^{2}+\ldots+z_{n}^{2}$. The Lie norm is equal to the so-called projective crossnorm $\|z\|_{\wedge}$ for the projective tensor product $\mathbb{R}^{n} \widehat{\otimes}_{\mathbb{R}} \mathbb{C}$ (here $\mathbb{R}^{n}$ is understood to be the Euclidean space with its canonical inner product and norm). One can easily prove that in general we have the inequality

$$
\Psi_{B}(z) \geq\|z\|_{\wedge}, \quad z \in \mathbb{C}^{n} .
$$

Here

$$
\|z\|_{\wedge}=\inf \left\{\sum_{j=1}^{m}\left|\alpha_{j}\right| q\left(x_{j}\right): z=\sum_{j=1}^{m} \alpha_{j} x_{j}, \alpha_{j} \in \mathbb{C}, x_{j} \in \mathbb{R}^{n}\right\}
$$

is a norm in $X \widehat{\otimes}_{\mathbb{R}} \mathbb{C}$, where $X=\left(\mathbb{R}^{n}, q\right)$ is a normed space such that $B=\left\{x \in \mathbb{R}^{n}: q(x) \leq 1\right\}$. A few years ago Professor Siciak posed the question of whether in $(*)$ one has equality. In particular, is this true for the square $B=[-1,1] \times[-1,1]$ ?

In this paper, we show that, in general, equality in $(*)$ cannot hold for all $z \in \mathbb{C}^{n}$. This is a corollary to Theorem 2.3 where explicit formulas are given for $\Psi_{B}$ for a wide family of norms in $\mathbb{R}^{2}$. The main goal of this paper is to show a relation between the extremal function $\Psi_{B}$, where $B$ is a ball in $\mathbb{R}^{2}$ with respect to a norm $q$, and the Cauchy-Poisson transform which is an important tool in harmonic analysis (see [St], [SW])). Note that for $x \in \mathbb{R}^{n}$ one has

In particular,

$$
\Psi_{B}(x)=q(x)
$$

$$
\log \Psi_{B}(1, t)=\log q(1, t)
$$

if $q$ is a norm in $\mathbb{R}^{2}$. Starting from the above fact, we show how to get an integral representation for $\Psi_{B}$. At the end of the paper we extend our result to a wider family of sets.

Acknowledgements. This paper was written during the author's stay at the Emile Picard Laboratory of the Paul Sabatier University of Toulouse in the academic year 1996/97. The author would like to express his gratitude 
to this Laboratory for excellent working conditions and personally to Dr. Jean Paul Calvi for his assistance and hospitality.

1. Cauchy-Poisson transform. Let $\mathbb{H}_{+}$and $\mathbb{H}_{-}$be the upper and lower halfplanes, respectively. If $q$ is a norm in $\mathbb{R}^{2}$, we put $u(t)=\log q(1, t)$. We denote by $\mathcal{P} u$ the Cauchy-Poisson transform of $u$ in $\mathbb{H}_{+}$(see e.g. [St]):

$$
\mathcal{P} u(\zeta)=(\Im \zeta) \frac{1}{\pi}_{-\infty}^{\infty}|\zeta-t|^{-2} u(t) d t=\frac{1}{\pi}_{-\infty}^{\infty} u(t y+x) \frac{d t}{1+t^{2}},
$$

where $\zeta=x+i y \in \mathbb{H}_{+}$.

LEMmA 1.1. If $0<\alpha<1$ then there exists a constant $C=C(\alpha)$ such that for $x, x^{\prime} \in \mathbb{R}$ and $y>0$ we have

$$
\left|\mathcal{P} u(\zeta)-u\left(x^{\prime}\right)\right| \leq C\left\{\left|x-x^{\prime}\right|+y\right\}^{\alpha}, \quad \zeta=x+i y .
$$

Proof. Observe that for $t, \tau \in \mathbb{R}$ we have

$$
\begin{aligned}
|\log q(1, t)-\log q(1, \tau)| & \leq M_{\alpha}\left[|q(1, t)-q(1, \tau)|(\min \{q(1, t), q(1, \tau)\})^{-1}\right]^{\alpha} \\
& \leq M_{\alpha}\left[q(0,1)|t-\tau|(\min \{q(1, t), q(1, \tau)\})^{-1}\right]^{\alpha} \\
& \leq M_{\alpha}\left[\frac{q(0,1)}{\inf _{t \in \mathbb{R}} q(1, t)}\right]^{\alpha}|t-\tau|^{\alpha}=M_{\alpha}^{\prime}|t-\tau|^{\alpha}
\end{aligned}
$$

where $M_{\alpha}=\sup _{x>0}(\log (1+x)) / x^{\alpha}$. Now we have

$$
\begin{aligned}
\left|\mathcal{P} u(\zeta)-u\left(x^{\prime}\right)\right| & \leq \frac{1}{\pi}_{-\infty}^{\infty}\left|u(t y+x)-u\left(x^{\prime}\right)\right| \frac{d t}{1+t^{2}} \\
& \leq \frac{M_{\alpha}^{\prime}}{\pi}{ }_{-\infty}^{\infty}\left|t y+x-x^{\prime}\right|^{\alpha} \frac{d t}{1+t^{2}} \\
& \leq \frac{M_{\alpha}^{\prime}}{\pi}{ }_{-\infty}^{\infty} \frac{(1+|t|)^{\alpha}}{1+t^{2}} d t\left[\left|x-x^{\prime}\right|+y\right]^{\alpha}=C(\alpha)\left[\left|x-x^{\prime}\right|+y\right]^{\alpha},
\end{aligned}
$$

which completes the proof.

COROllary 1.2. The function $\mathcal{P} u$ extends to a continuous function in $\overline{\mathbb{H}}_{+}$that is harmonic in $\mathbb{H}_{+}$. If we set

$$
\mathcal{P} u(\zeta)=\mathcal{P} u(\bar{\zeta}), \quad \zeta \in \mathbb{H}_{-},
$$

we obtain a continuous function in $\mathbb{C}$, symmetric with respect to the real axis and harmonic in $\mathbb{H}_{+} \cup \mathbb{H}_{-}$. Moreover, for $\zeta=x+i y$, we have

$$
\mathcal{P} u(\zeta)=\frac{1}{\pi}_{-\infty}^{\infty} u(t|y|+x) \frac{d t}{1+t^{2}}, \quad \zeta \in \mathbb{C} .
$$


Applying the maximum principle for subharmonic functions in $\mathbb{H}_{+}$or $\mathbb{H}_{-}$, we easily obtain the following important

Corollary 1.3. If $B=\left\{x \in \mathbb{R}^{2}: q(x) \leq 1\right\}$ then

$$
\log \Psi_{B}(1, \zeta) \leq \mathcal{P} u(\zeta), \quad \zeta \in \mathbb{C} .
$$

Now we prove that $\mathcal{P} u \in \mathcal{S H}(\mathbb{C})$. To do this we need the following results which are interesting in themselves.

For a fixed $\alpha \in(-1,1)$, define

$$
v(\alpha, y):=\frac{1}{2} \log \left(1+2 \alpha y+y^{2}\right), \quad y \in \mathbb{R},
$$

and set $\beta=\sqrt{1-\alpha^{2}}$. Note that if $|y|<1$ then

$$
v(-\alpha, y)=-\sum_{k=1}^{\infty} \frac{1}{k} T_{k}(\alpha) y^{k}
$$

where $T_{k}(\alpha)$ denotes the $k$ th Chebyshev polynomial $T_{k}(\alpha)=\cos (k \arccos \alpha)$ (see e.g. $[\mathrm{SW}])$.

LEMma 1.4. For all $y \in \mathbb{R}$,

$$
\frac{1}{\pi}_{-\infty}^{\infty} v(\alpha, t y) \frac{d t}{1+t^{2}}=v(\beta,|y|) .
$$

Pr o of. Denote the left hand side of the above formula by $F_{\alpha}(y)$. Since $F_{\alpha}(y)$ and $v(\beta,|y|)$ are even functions that agree at 0 , it suffices to show that $F_{\alpha}^{\prime}(y)=v^{\prime}(\beta, y)$ for $y>0$. We can check this by applying the residue method. The calculation is rather simple but a little laborious so we omit it.

LEMMA 1.5. If $\zeta=x+i y$ then

$$
\mathcal{P} v(\alpha, \zeta)=\frac{1}{\pi}{ }_{-\infty}^{\infty} v(\alpha, t|y|+x) \frac{d t}{1+t^{2}}=\frac{1}{2} \log \left(1+2 \alpha x+x^{2}+2 \beta|y|+y^{2}\right) .
$$

Proof. We apply Lemma 1.4 with

$$
\alpha^{\prime}=\frac{\alpha+x}{\sqrt{1+2 \alpha x+x^{2}}} \quad \text { and } \quad y^{\prime}=\frac{|y|}{\sqrt{1+2 \alpha x+x^{2}}} .
$$

LEMMA 1.6. $\mathcal{P} v(\alpha, \zeta) \in \mathcal{S H}(\mathbb{C})$.

Pro of. We apply the Zaremba criterion (see [L, pp. 439-440]). Let $v \in$ $\mathcal{C}(\Omega)$. Put

$$
\Delta_{h} v(\zeta)=v(\zeta+h)+v(\zeta-h)+v(\zeta+i h)+v(\zeta-i h)-4 v(\zeta), \quad h \in \mathbb{R}_{*},
$$

and define the Zaremba operator

$$
\underline{\Delta v}(\zeta):=\limsup _{h \rightarrow 0} \frac{1}{h^{2}} \Delta_{h} v(\zeta) .
$$


Then $v \in \mathcal{S H}(\Omega)$ iff $\underline{\Delta} v \geq 0$ in $\Omega$. We apply this criterion to $\mathcal{P} v(\alpha, \zeta)$. If $\zeta \in \mathbb{C} \backslash \mathbb{R}$ then $\Delta \mathcal{P} v(\alpha, \zeta)=\Delta \mathcal{P} v(\alpha, \zeta)=0$, since $\mathcal{P} v(\alpha, \zeta)$ is harmonic in $\mathbb{C} \backslash \mathbb{R}$. If $\zeta \in \mathbb{R}$, we easily calculate that $\underline{\mathcal{P}} v(\alpha, \zeta)=\infty$.

Corollary 1.7. Let $u(t)=\frac{1}{2} \log \left(a t^{2}+b t+c\right)$ and $\alpha=b /(2 \sqrt{a c})$, where $\Delta=b^{2}-4 a c<0, c, a>0$. Then $\mathcal{P} u \in \mathcal{S H}(\mathbb{C})$.

Proof. We have

$$
\mathcal{P} u(\zeta)=\mathcal{P} v\left(\alpha, \sqrt{\frac{a}{c}} \zeta\right)+\frac{1}{2} \log c,
$$

whence we can apply Lemma 1.6.

Now we are in a position to prove the following

Proposition 1.8. If $q$ is a norm in $\mathbb{R}^{2}$ and $u(t)=\log q(1, t)$, then $\mathcal{P} u \in \mathcal{S H}(\mathbb{C})$. This implies that $\mathcal{P} u$ belongs to the Lelong class $\mathcal{L}(\mathbb{C})$.

Proof. Denote by $q^{*}$ the dual norm: $q^{*}(x)=\sup \{x \cdot y: y \in B\}$. We can write (see [B3])

$$
q(x)=\sup \left\{x \cdot y / q^{*}(y): y \in S^{1}\right\}=\lim _{k \rightarrow \infty} q_{k}(x),
$$

where $q_{k}(x)=\left[{ }_{S^{1}}\left(x \cdot y / q^{*}(y)\right)^{2 k} d \sigma(y)\right]^{1 / 2 k}$ is a (smooth) norm in $\mathbb{R}^{2}$ and $q_{k}^{2 k}$ is a polynomial of degree $2 k$. Moreover (cf. [B3] again), the sequence $q_{k}$ is increasing. Thus $q_{k}^{2 k}(1, \zeta)$ is a polynomial of degree $2 k$ with real coefficients and without any real zeros. Applying Corollary 1.7 we easily check that $\mathcal{P} u_{k} \in \mathcal{S H}(\mathbb{C})$, where $u_{k}(t)=\log q_{k}(1, t)$. Finally, we have

$$
\begin{aligned}
\mathcal{P} u(\zeta) & =\lim _{k \rightarrow \infty} \mathcal{P} u_{k}(\zeta) \leq \lim _{k \rightarrow \infty} \frac{1}{2 \pi}{ }_{-\pi}^{\pi} \mathcal{P} u_{k}\left(\zeta+r e^{i \theta}\right) d \theta \\
& \leq \frac{1}{2 \pi}_{-\pi}^{\pi} \mathcal{P} u\left(\zeta+r e^{i \theta}\right) d \theta,
\end{aligned}
$$

which completes the proof.

2. Homogeneous extremal function for a ball in $\mathbb{R}^{2}$. The main result of this paper is the following

Theorem 2.1. If $q$ is a norm in $\mathbb{R}^{2}, B=\left\{x \in \mathbb{R}^{2}: q(x) \leq 1\right\}$ and $u(t)=\log q(1, t), t \in \mathbb{R}$, then

$$
\Psi_{B}(1, \zeta)=\exp \mathcal{P} u(\zeta), \quad \zeta \in \mathbb{C} .
$$

Consequently,

$$
\Psi_{B}\left(z_{1}, z_{2}\right)=\left|z_{1}\right| \exp \mathcal{P} u\left(z_{2} / z_{1}\right) .
$$


Proof. We know that $\log \Psi_{B}(1, \zeta) \leq \mathcal{P} u(\zeta), \zeta \in \mathbb{C}$. To prove the opposite inequality, define

$$
\phi(\zeta, z)=\left\{\begin{array}{l}
|\zeta| \exp \mathcal{P} u\left(\zeta^{-1} z\right), \quad \zeta \in \mathbb{C}_{*}, \quad z \in \mathbb{C}, \\
\limsup _{\xi \rightarrow 0, \xi \neq 0}|\xi| \exp \mathcal{P} u\left(\xi^{-1} z\right), \quad \zeta=0, \quad z \in \mathbb{C}
\end{array}\right.
$$

(cf. [Kl, proof of Thm. 5.1.6]). Then $\phi \in \exp \mathcal{L}\left(\mathbb{C}^{2}\right)$ and $\phi(\zeta w)=|\zeta| \phi(w)$, $\phi_{\mid B} \leq 1$. This means that

$$
\phi(\zeta, z) \leq \Psi_{B}(\zeta, z)
$$

whence $\mathcal{P} u(\zeta) \leq \log \Psi_{B}(1, \zeta)$. This completes the proof.

As an interesting application, we prove the following result on a harmonic foliation related to the extremal function $\log \Psi_{B}$. A similar foliation is related to the extremal function $V_{B}=\log \Phi_{B}$ (see [B1], [B2] for details).

Corollary 2.2. Let $X=\left(\mathbb{R}^{2}, q\right)$, let $\check{X}=X \check{\otimes}_{\mathbb{R}} \mathbb{C}$ be the injective tensor product, and let $\check{S}$ be the unit sphere in $\check{X}$. Define

$$
\chi(\zeta, c)=\frac{1}{2}\left(\zeta c+\zeta^{-1} \bar{c}\right), \quad \zeta \in \mathbb{D}^{*}=\mathbb{C} \backslash \overline{\mathbb{D}}, c \in \check{S} .
$$

Then $\log \Psi_{B}$ is harmonic on each leaf $\chi(\zeta, c), c \in \check{S}$.

Proof. Let $\chi(\zeta, c)=\left(\chi_{1}(\zeta, c), \chi_{2}(\zeta, c)\right)$, where $c=a+i b$. Then $\chi_{j}(\zeta, c)$ $=g(\zeta) a_{j}+i \widehat{g}(\zeta) b_{j}, j=1,2$, with $g(\zeta)=\frac{1}{2}\left(\zeta+\zeta^{-1}\right)$ and $\widehat{g}(\zeta)=\frac{1}{2}(\zeta-$ $\left.\zeta^{-1}\right)$. Without loss of generality we can assume that $c_{1} \neq 0$ and $\operatorname{det}(a, b)=$ $\operatorname{det}\left(\left(a_{1}, a_{2}\right),\left(b_{1}, b_{2}\right)\right) \neq 0$. Then we can write

$$
\log \Psi_{B}(\chi(\zeta, c))=\log \left|\chi_{1}(\zeta, c)\right|+\mathcal{P} u\left(\chi_{2}(\zeta, c) / \chi_{1}(\zeta, c)\right) .
$$

Now observe that the mapping

$$
\phi_{c}(\zeta)=\chi_{2}(\zeta, c) / \chi_{1}(\zeta, c): \mathbb{D}^{*} \rightarrow \mathbb{C}
$$

takes its values in $\mathbb{H}_{+}$or in $\mathbb{H}_{-}$. Indeed, we have

$$
\phi_{c}(\zeta)=\left|\chi_{1}(\zeta, c)\right|^{-2} \chi_{2}(\zeta, c) \overline{\chi_{1}(\zeta, c)}
$$

and

$$
\Im\left(\chi_{2}(\zeta, c) \overline{\chi_{1}(\zeta, c)}\right)=\frac{1}{4} \operatorname{det}(a, b)\left(|\zeta|^{2}-|\zeta|^{-2}\right),
$$

whence $\operatorname{sgn} \Im\left(\phi_{c}(\zeta)\right)$ is constant in $\mathbb{D}^{*}$. Therefore $\mathcal{P} u\left(\phi_{c}(\zeta)\right)$ is a harmonic function as a composition of a harmonic function with a holomorphic one.

Applying Lemmas 1.5, 1.6 and Theorem 2.1 we can explicitly calculate $\Psi_{B}$ for an important class of norms.

THEOREM 2.3. If $n$ is a fixed natural number, $q_{n}(x)=\left(x_{1}^{2 n}+x_{2}^{2 n}\right)^{1 /(2 n)}$ and $S_{n}=\left\{x \in \mathbb{R}^{2}: q_{n}(x)=1\right\}$, then, for all $z \in \mathbb{C}^{2}$, 


$$
\Psi_{S_{n}}(z)=\left[\prod_{j=1}^{n}\left(\left|z_{1}\right|^{2}-2 \alpha_{j} \Re\left(z_{1} \bar{z}_{2}\right)+\left|z_{2}\right|^{2}+2\left|\beta_{j}\right|\left|\Im\left(z_{1} \bar{z}_{2}\right)\right|\right)^{1 / 2}\right]^{1 / n},
$$

where $\zeta_{j}=\alpha_{j}+i \beta_{j} \in \sqrt[2 n]{-1}, j=1, \ldots, n$, with $\zeta_{j} \neq \bar{\zeta}_{k}$ for $j \neq k$.

Corollary 2.4. If $q_{\infty}(x)=\max \left(\left|x_{1}\right|,\left|x_{2}\right|\right)$ and $S_{\infty}=\left\{x \in \mathbb{R}^{2}: q_{\infty}(x)\right.$ $=1\}$, then for all $z \in \mathbb{C}^{2}$,

$\Psi_{S_{\infty}}(z)=\exp \left[{ }_{0}^{2 \pi} \log \left(\left|z_{1}\right|^{2}-2 \cos \theta \Re\left(z_{1} \bar{z}_{2}\right)+\left|z_{2}\right|^{2}+2\left|\sin \theta \Im\left(z_{1} \bar{z}_{2}\right)\right|\right)^{1 / 2} \frac{d \theta}{2 \pi}\right]$.

Proof of Theorem 2.3. Fix an $n \in \mathbb{N}$. We have

$$
1+\zeta^{2 n}=\prod_{j=1}^{n}\left(\zeta-\zeta_{j}\right)\left(\zeta-\bar{\zeta}_{j}\right)=\prod_{j=1}^{n}\left(1-2 \alpha_{j} \zeta+\zeta^{2}\right) .
$$

Consider $u_{n}(t)=(2 n)^{-1} \log \left(1+t^{2 n}\right)=\log f_{n}(t)$, where $f_{n}(t)=q_{n}(1, t)$. Applying Lemma 1.5 and $(*)$ we obtain

$$
(* *) \quad \mathcal{P} u_{n}(\zeta)=\frac{1}{2 n} \sum_{j=1}^{n} \log \left(1-2 \alpha_{j} \Re \zeta+|\zeta|^{2}+2\left|\beta_{j}\right||\Im \zeta|\right) .
$$

By Theorem 2.1 we have $\Psi_{S_{n}}(1, \zeta)=\exp \mathcal{P} u_{n}(\zeta)$, whence, by homogeneity of $\Psi$,

$$
\Psi_{S_{n}}\left(z_{1}, z_{2}\right)=\left|z_{1}\right| \exp \mathcal{P} u_{n}\left(z_{2} \bar{z}_{1}\left|z_{1}\right|^{-2}\right),
$$

and applying $(* *)$ we get the formula of Theorem 2.3.

REMARK 2.5. If $B$ is the unit ball and $S$ is the unit sphere for a norm $q$ in $\mathbb{R}^{2}$ then $\mathbb{T} B$ and $\mathbb{T} S$, where $\mathbb{T}$ is the unit circle in $\mathbb{C}$, are circular subsets of $\mathbb{C}^{2}$. Hence we obtain

$$
\Phi_{\mathbb{T} S}(z)=\max \left(1, \Psi_{\mathbb{T} S}(z)\right)=\max \left(1, \Psi_{B}(z)\right), \quad z \in \mathbb{C}^{2} .
$$

Let $X=\left(\mathbb{R}^{2}, q\right), \widehat{X}=X \widehat{\otimes}_{\mathbb{R}} \mathbb{C}$ and let $\widehat{B}$ be the unit (closed) ball in $\widehat{X}$. It is well known that

$$
\operatorname{extr} \widehat{B}=\left\{e^{i \theta} x: x \in \operatorname{extr} B, \theta \in[-\pi, \pi]\right\}=\mathbb{T} \operatorname{extr} B .
$$

In particular, if $X$ is a strictly convex space then

$$
\operatorname{extr} \widehat{B}=\mathbb{T} S .
$$

Hence we get the following 
COROllary 2.6. If $\left(\mathbb{R}^{2}, q\right)$ is a strictly convex space then

$$
\Phi_{\operatorname{extr} \widehat{B}}(z)=\max \left(1, \Psi_{B}(z)\right)=\max \left(1,\left|z_{1}\right| \exp \mathcal{P} u\left(z_{2} / z_{1}\right)\right), \quad z \in \mathbb{C}^{2},
$$

where $u(t)=\log q(1, t)$.

COROLlaRY 2.7. If $q$ is a norm in $\mathbb{R}^{2}, S$ is its unit sphere and $u(t)=$ $\log q(1, t)$, then

$$
\widehat{\mathbb{T} S}=\left\{z \in \mathbb{C}^{2}: \log \left|z_{1}\right|+\mathcal{P} u\left(z_{2} / z_{1}\right) \leq 0\right\},
$$

where $\widehat{K}$ denotes the polynomially convex hull of $K$.

Note that the equality $\Psi_{B}(z)=\|z\|_{\wedge}$ is equivalent to

$$
\widehat{\mathbb{T} S}=\operatorname{conv}(\mathbb{T} S) \text {. }
$$

In particular, if $(X, q)$ is a strictly convex space then $\Psi_{B}(z)=\|z\|_{\wedge}$ iff $(\operatorname{extr} \widehat{B})=\widehat{B}$.

REMARK 2.8. Theorem 2.1 can be extended in the following way. Denote by $\Gamma_{0}$ the class of all continuous, nonnegative and absolutely homogeneous functions $g$ on $\mathbb{R}^{2}$ (i.e. $g(t x)=|t| g(x), t \in \mathbb{R}, x \in \mathbb{R}^{2}$ ) such that $g$ has the form

$$
g(x)=\max _{1 \leq k \leq n} Q_{k}(x)^{1 / \operatorname{deg} Q_{k}},
$$

where $Q_{k} \geq 0$ are homogeneous polynomials and $Q_{1}^{-1}(0)=\{0\}$. Denote by $\Gamma$ the class of continuous, nonnegative and homogeneous functions $g$ with $g^{-1}(0)=\{0\}$ which are generated by $\Gamma_{0}$ with respect to the operations: limit of monotonic sequences and $\left(g_{1} \cdot \ldots \cdot g_{N}\right)^{1 / N}$. We show that Theorem 2.1 extends to $\Gamma$. We need the following

LEMmA 2.9. If $g \in \Gamma_{0}$ and $u(t)=\log g(1, t)$ then $\mathcal{P} u$ is a continuous function that belongs to $\mathcal{L}(\mathbb{C})$.

Pr o of. The proof that $\mathcal{P} u$ is continuous is similar to that of Lemma 1.1. It is easily seen that all numbers $\operatorname{deg} Q_{j}$ are even. Put $N=\operatorname{deg} Q_{1} \cdot \ldots \cdot \operatorname{deg} Q_{n}$ and define

$$
q_{k}(x)=\frac{1}{n}\left(Q_{1}(x)^{2 k N / \operatorname{deg} Q_{1}}+\ldots+Q_{n}(x)^{2 k N / \operatorname{deg} Q_{n}}\right) .
$$

Then $q_{k}$ is a sequence of homogeneous polynomials of degree $2 k N$ and the sequence $g_{k}=q_{k}^{1 /(2 k N)}$ increases to $g$. Let $u_{k}(t)=\log g_{k}(1, t)$. Applying Corollary 1.7 we easily obtain $\mathcal{P} u_{k} \in \mathcal{S H}(\mathbb{C})$. Hence, similarly to the proof of Proposition 1.8, we show that $\mathcal{P} u \in \mathcal{S H}(\mathbb{C})$ and therefore $\mathcal{P} u \in \mathcal{L}(\mathbb{C})$.

Corollary 2.10. Let $g \in \Gamma$ and let $u(t)=\log g(1, t)$. Then $\mathcal{P} u \in \mathcal{L}(\mathbb{C})$.

Now one can easily obtain a generalization of Theorem 2.1; its proof is left to the reader. 
TheOREM 2.11. Let $g \in \Gamma$ and let $B=\left\{x \in \mathbb{R}^{2}: g(x) \leq 1\right\}$. Set $u(t)=\log g(1, t)$. Then

$$
\Psi_{B}\left(z_{1}, z_{2}\right)=\left|z_{1}\right| \exp \mathcal{P} u\left(z_{2} / z_{1}\right) .
$$

Corollary 2.12. For $g_{1}, \ldots, g_{n} \in \Gamma$, put $B_{j}=\left\{x \in \mathbb{R}^{2}: g_{j}(x) \leq 1\right\}$. Define also $g(x)=\left(g_{1} \cdot \ldots \cdot g_{n}\right)^{1 / n}$ and $B=\left\{x \in \mathbb{R}^{2}: g(x) \leq 1\right\}$. Then

$$
\Psi_{B}=\left(\Psi_{B_{1}} \cdot \ldots \cdot \Psi_{B_{n}}\right)^{1 / n} .
$$

Corollary 2.13. Let $g \in \Gamma$ and let $S=\left\{x \in \mathbb{R}^{2}: g(x)=1\right\}$. If $u(t)=\log g(1, t)$ then

$$
\Phi_{\mathbb{T} S}\left(z_{1}, z_{2}\right)=\max \left(1,\left|z_{1}\right| \exp \mathcal{P} u\left(z_{2} / z_{1}\right)\right)
$$

and

$$
\widehat{\mathbb{T} S}=\left\{z \in \mathbb{C}^{2}: \log \left|z_{1}\right|+\mathcal{P} u\left(z_{2} / z_{1}\right) \leq 0\right\}
$$

\section{References}

[B1] M. Baran, Siciak's extremal function of convex sets in $\mathbb{C}^{n}$, Ann. Polon. Math. 48 (1988), 275-280.

[B2] - Plurisubharmonic extremal functions and complex foliations for the complement of convex sets in $\mathbb{R}^{n}$, Michigan Math. J. 39 (1992), 395-404.

[B3] - Complex equilibrium measure and Bernstein type theorems for compact sets in $\mathbb{R}^{n}$, Proc. Amer. Math. Soc. 123 (1995), 485-494.

[B4] -, Bernstein type theorems for compact sets in $\mathbb{R}^{n}$ revisited, J. Approx. Theory 69 (1992), 156-166.

[CL] E. W. Cheney and W. A. Light, Approximation Theory in Tensor Product Spaces, Lecture Notes in Math. 1169, Springer, Berlin, 1985.

[D] L. M. Drużkowski, Effective formula for the crossnorm in complexified unitary spaces, Univ. Iagel. Acta Math. 16 (1974), 47-53.

[Kl] M. Klimek, Pluripotential Theory, Oxford Univ. Press, 1991.

[L] F. Leja, Teoria funkcji analitycznych [Theory of Analytic Functions], PWN, Warszawa, 1957 (in Polish).

[Si1] J. Siciak, On an extremal function and domains of convergence of series of homogeneous polynomials, Ann. Polon. Math. 25 (1961), 297-307.

[Si2] - On some extremal functions and their applications in the theory of analytic functions of several complex variables, Trans. Amer. Math. Soc. 105 (1962), 322357.

[Si3] -, Holomorphic continuation of harmonic functions, Ann. Polon. Math. 29 (1974), 67-73.

[Si4] -, Extremal plurisubharmonic functions in $\mathbb{C}^{n}$, ibid. 39 (1981), 175-211.

[Si5] -, Extremal Plurisubharmonic Functions and Capacities in $\mathbb{C}^{n}$, Sophia Kokyuroku in Math. 14, Sophia Univ., Tokyo, 1982. 
[St] E. M. Stein, Singular Integrals and Differentiability Properties of Functions, Princeton Univ. Press, 1970.

[SW] E. M. Stein and G. Weiss, Introduction to Fourier Analysis on Euclidean Spaces, Princeton Univ. Press, 1971.

Institute of Mathematics

Jagiellonian University

Reymonta 4

30-059 Kraków, Poland

E-mail: baran@im.uj.edu.pl

Reçu par la Rédaction le 27.11.1997

Révisé le 9.10.1998 